\title{
RNA at Santa Cruz
}

Nestled in an atmosphere that resembles a national park, the Center for Molecular Biology of RNA at the University of California, Santa Cruz is fulfilling Harry Noller's vision of bringing together multidisciplinary researchers to solve problems that range from finding obscure RNAs to understanding the origin of life.

In a postgenomics world, we need to know "how it all comes together," according to Sofie Salama of UCSC's RNA Center. How it has all come together is a recurring theme not only of the research but also of the history of the seven labs that are officially affiliated with the RNA Center. For most of the past 14 years, Harry Noller's vision as the head of the Center has been to promote research at the interface of such diverse fields as computational biology, structural biology, genetics, biochemistry and molecular biology. Thus it comes as no surprise that, as indicated by Melissa Jurica, the newest faculty member in the Center, what brings everybody together is a single molecule: RNA.

At its beginning, the Center found itself at the finish line of the human genome sequencing project, with the assembly of the genome taking place in a bank of computers that has now expanded into a "pitacluster." The pitacluster is a testament to the amount of data that is being processed not only through the Center's public browsers (http://genome.ucsc.edu/, http://archaea.ucsc.edu) but also in finding new RNAs, as in Todd Lowe's group. Indeed, with the emergence of so many types of RNAs (for example, small interfering RNAs), it is not surprising that much of the emphasis of the Center is on computational approaches that can detect the next new RNA class. Because Lowe believes that "the more you connect model organisms to closely related species, the more useful the model is," he recently cataloged huge numbers of new RNAs from the genomes of his favorite critters, the archaebacteria. He is currently working with William Scott to characterize one of them structurally.

Another striking example of this kind of comparative analysis is the case of an unusual and unprecedented noncoding RNA called Harl that was found by David Haussler's group (Nature 443, 167-172, 2006). This RNA is tremendously conserved in mammals all the way up to the chimpanzee, but the group found dramatic substitutions between the sequences in chimpanzees and humans. These differences may reveal new ways in which human brains are distinct from those of other mammals. Haussler's group has also been pursuing the role of a conserved structural element in the 3' terminus of all coronavirus mRNAs. On account of the infrastructure of the Center, which combines the computational expertise of Haussler and Lowe with molecular biologists like Alan Zahler and Manuel Ares, the project of first finding this motif on the computer and then solving its structure took a mere six months. Salama, who runs Haussler's "wet" lab, is proud to note that contrary to popular belief, "the wet lab is not necessarily the rate-limiting step" when it comes to solving problems. This is probably a result not of expedient experimentalists but instead of the increasing use of computational approaches. Indeed, in a time when new classes of RNAs are being found every year, and with new roles in molecular biology and developmental biology attributable to these RNAs, it is clear that the computational approaches are increasingly important, yet labor intensive.

Additional structural efforts stem from Noller's group, which is doing everything possible to study the ribosome and how it functions and changes during translation. Indeed, the Center has played an integral part in solving the structure of the ribosome by crystallography, which remains the largest asymmetric molecular structure ever solved. What has impressed Noller the most in his recent studies monitoring individual ribosome movements by fluorescence resonance energy transfer (FRET) is that "it's not just like an automobile engine with rigid parts, but the whole thing is alive and pulsing." With bittersweet sentiment, Noller recalls a time nearly 25 years ago when his proposal that rRNA is functional and not merely a scaffold for ribosomal proteins was labeled as a "crackpot" idea by distinguished colleagues.

Looking at a different living machine, Scott's group recently published a new crystal structure of the full-length hammerhead ribozyme that has changed the common view of the chemical mechanism of this RNA (Cell 126, 309-320, 2006). Similarly, Jurica uses X-ray crystallography and cryoelectron microscopy to study one of the numerous RNA-containing machines, the spliceosome. Fueled by these and other reports of functional RNA, scientists have continued looking for important RNA sequences, and the numerous RNA machines that now exist provide the Center and their collaborators with a plethora of research directions. These range

"[The ribosome is] not
just like an automobile
engine with rigid parts,
but the whole thing is
alive and pulsing."

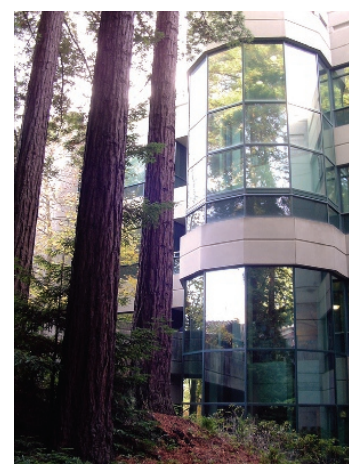

from finding obscure RNAs (which, because so much of the genome's transcribed RNA is ultimately not translated, turns out to be the norm rather than the exception) to gaining insights into the origin of life.

Noller notes, "there have been so many surprises just within the last year, it looks like that may be the toe of a very steep curve." Looking to the future, another goal that intrigues the Center (beyond cataloging unique RNAs and their functions) is understanding the origin of life. According to Noller, there is "almost a quasi-religious aspect of working on RNA in that it might tell you where we came from." He adds that "the discovery of the ribozyme solved the chicken-and-egg problem of life" in that researchers can now provide a plausible model that takes into account the requirement for both an information-coding molecule and a catalyst.

The interdisciplinary work being carried out at the UCSC RNA Center creates a stimulating environment for doing research, even if interdisciplinary work can be difficult because of diverse backgrounds, as Salama notes. She believes that their group, which doesn't have a history of RNA biology, has been successful because of the support they have received from colleagues in the Center. Combined with the remarkable facilities, the diverse backgrounds afford the Center the unique ability to test models with exquisite precision. According to Noller, "it's always a question of maximizing signal to noise; one way is increasing your signal, or you can decrease the noise." This latter strategy is certainly revealed when you visit the Center and look out to the redwood trees that dominate the campus landscape. Being shielded from congestion and a high-pressure atmosphere, Noller believes that the Center has tackled scientific problems that wouldn't have been possible if they had been situated in a more typical big university environment.

Mirella Bucci, Santa Cruz, California 